Article

\title{
Differences in the Regulation of Ochratoxin A by the HOG Pathway in Penicillium and Aspergillus in Response to High Osmolar Environments
}

\author{
Dominic Stoll, Markus Schmidt-Heydt and Rolf Geisen *
}

Department of Safety and Quality of Fruits and Vegetables, Max Rubner-Institut, Haid-und-Neu-Str. 9, Karlsruhe 76121, Germany; E-Mails: dominic.stoll@mri.bund.de (D.S.);

markus.schmidt-heydt@mri.bund.de (M.S.-H.)

* Author to whom correspondence should be addressed; E-Mail: rolf.geisen@mri.bund.de;

Tel.: +49-721-6625-450; Fax: +49-721-6625-453.

Received: 17 May 2013; in revised form: 19 June 2013 / Accepted: 8 July 2013 /

Published: 19 July 2013

\begin{abstract}
Penicillium verrucosum, P. nordicum and Aspergillus carbonarius are three important ochratoxin A producing species. P. verrucosum is in addition able to produce citrinin. It has been shown earlier that $P$. nordicum is adapted to $\mathrm{NaCl}$ rich environments like salt rich dry cured foods or even salines. In this organism, the biosynthesis of ochratoxin A plays an adaptive role in this habitat. $P$. verrucosum generally can be found on cereals, but occasionally also on salt rich dry cured foods. In contrast $A$. carbonarius usually cannot be found in $\mathrm{NaCl}$ rich environments, but it occurs in another environment with high concentration of solutes, e.g., in sugar rich substrates like grapes and grape juices. Usually osmotic challenging conditions activate the HOG MAP kinase signal cascade, which in turn activates various osmo-regulated genes. In the current analysis, it could be demonstrated that in case of $P$. nordicum and $P$. verrucosum the $\mathrm{NaCl}$ induced production of ochratoxin A is correlated to the phosphorylation status of the HOG MAP kinase. Just the opposite was true for A. carbonarius. In this case, also higher amounts of $\mathrm{NaCl}$ in the medium lead to an increased phosphorylation status of HOG, but no increase in ochratoxin biosynthesis was observed. In contrast to the Penicillia, higher $\mathrm{NaCl}$ concentrations lead to a rapid cessation of growth by A. carbonarius. High glucose concentrations have much less impact on growth and the phosphorylation of HOG.
\end{abstract}


Keywords: Penicillium nordicum; Penicillium verrucosum; Aspergillus carbonarius; ochratoxin; osmotic stress; HOG signaling cascade

Abbreviations: YES: yeast extract sucrose medium; MEA: malt extract agar; HOG signal pathway: high osmolarity glycerol signal pathway; MAPK: mitogen activated protein kinase; ATMT: Agrobacterium tumefaciens mediated transformation; TLC: thin layer chromatography

\section{Introduction}

Ochratoxin A is a nephrotoxic mycotoxin produced by some Aspergillus and Penicillium species. Because of the temperature and humidity preferences ochratoxigenic Aspergilli occur mainly in regions with elevated temperatures and occur on food commodities like coffee (A. westerdijkiae, A. steynii, A. ochraceus) [1], dried fruits (A. carbonarius, A. ochraceus) [2], spices (A. ochraceus) [3] or grapes (A. carbonarius) [4-6]. On the other hand, the Penicillia are adapted to regions with moderate climate because of their lower temperature growth optimum. $P$. verrucosum can mainly be found on cereals and is responsible for the occurrence of ochratoxin A in cereal-based products [7]. $P$. nordicum often occurs in $\mathrm{NaCl}$ rich environments mainly in dry cured meats or cheeses but may even be found in pure $\mathrm{NaCl}$ [8-10]. Despite the fact that $P$. nordicum is the main ochratoxigenic species in $\mathrm{NaCl}$ rich environments, occasionally also $P$. verrucosum can be identified in these commodities [11-13]. $P$. nordicum is a very consistent and high ochratoxin A producing species. A. carbonarius also produces elevated amounts of ochratoxin A, albeit usually not at the level of P. nordicum. In contrast, $P$. verrucosum generally produces only moderate amounts of ochratoxin A. In fact, the production of ochratoxin A by the latter species is highly dependent on the environmental conditions. $P$. verrucosum is also able to produce citrinin, predominantly under conditions of oxidative stress [14], whereas under high $\mathrm{NaCl}$ conditions the production of citrinin is shifted towards ochratoxin A [15]. It was recently been shown that the biosynthesis and excretion of ochratoxin A, which carries a chlorine in its molecule, can be regarded as a kind of adaptation to $\mathrm{NaCl}$ rich environments. The high production of ochratoxin $\mathrm{A}$ under high $\mathrm{NaCl}$ conditions by the Penicillia serves as a vehicle which pumps chlorine out of the cell and ensures a certain chlorine homeostasis, which increases the viability of the Penicillia under these conditions [15]. Because of the shift from citrinin towards ochratoxin A in P. verrucosum under $\mathrm{NaCl}$ conditions, also this species can, under certain circumstances, adapt to this environment and can indeed be found here [11-13]. In contrast to the two Penicillia, A. carbonarius was never reported to be found in $\mathrm{NaCl}$ rich environments. This indicates that $A$. carbonarius is not adapted to $\mathrm{NaCl}$ rich habitats, despite the fact that $A$. carbonarius is also able to produce ochratoxin A and should therefore be able to also use the mechanism described above. A. carbonarius however occurs in sugar rich environments like grapes, which maybe also osmotically challenging to the fungus.

Changes in the osmolarity of the medium, which can be caused by $\mathrm{NaCl}$ or sugars, are usually transmitted to the transcriptional level by the aid of signal cascades. The HOG signal cascade (high osmolarity glycerol) is the most important cascade for this environmental signal. This is a MAP kinase cascade with HOG as the last protein kinase. The phosphorylated form of HOG is the form that 
subsequently activates the downstream transcription factors. The involvement of this MAP kinase cascade in the regulation of mycotoxin biosynthesis has been shown for trichothecene biosynthesis by Fusarium graminearum [16] or alternariol biosynthesis in the case of Alternaria alternata [17].

Therefore, based on these conditions, the question arose whether the phosphorylation of HOG can be attributed to the regulation of the biosynthesis of ochratoxin A and whether there are differences in the regulation between the three species, which would explain the different adaptation to osmolar challenging conditions.

\section{Results}

2.1. Growth Behavior of P. nordicum, P. verrucosum and A. carbonarius on YES Medium with Increasing Concentrations of $\mathrm{NaCl}$

In order to analyze the influence of $\mathrm{NaCl}$ on growth and thereby on competitiveness of the three species under increasing $\mathrm{NaCl}$ conditions in the YES medium, the diameters of the colony were measured after growth at $25{ }^{\circ} \mathrm{C}$ for five days (Aspergillus) or for nine days (Penicillium) because of the differential growth rates. As can be seen in Figure 1 the colony diameters of the two Penicillia are very similar up to a $\mathrm{NaCl}$ concentration of $20 \mathrm{~g} / \mathrm{L}$. At higher $\mathrm{NaCl}$ concentrations growth of $P$. verrucosum decreased more with increasing $\mathrm{NaCl}$ concentrations than growth of $P$. nordicum. In fact at $80 \mathrm{~g} / \mathrm{L} \mathrm{NaCl}$ the colony diameter was less than half of that of the YES medium without $\mathrm{NaCl}$. $P$. nordicum in contrast showed a more adapted growth behavior. This species even showed a growth optimum at $40 \mathrm{~g} / \mathrm{L} \mathrm{NaCl}$ and above this concentration growth only ceases very slowly, reaching $85 \%$ of the growth at non-substituted YES medium at the highest $\mathrm{NaCl}$ concentration $(80 \mathrm{~g} / \mathrm{L} \mathrm{NaCl})$. In contrast to the Penicillia $A$. carbonarius is much more influenced by increasing $\mathrm{NaCl}$ concentrations. Already at $20 \mathrm{~g} / \mathrm{L} \mathrm{NaCl}$ growth is slightly decreased compared to the lower concentrations, however at concentrations beyond this value the growth capability of $A$. carbonarius reduces drastically with further increasing $\mathrm{NaCl}$ concentrations. At $80 \mathrm{~g} / \mathrm{L} \mathrm{NaCl}$ no growth at all could yet be identified after an incubation time of five days.

It has to be kept in mind that only the colony diameter is reported here, which of course is only one parameter for growth. From the morphological point of view the Penicillium colonies showed roughly the same morphology (sporulation, color, aerial density of the mycelium) at high $\mathrm{NaCl}$ concentrations than the colonies grown at non-modified medium. This was not the case for $A$. carbonarius. At higher $\mathrm{NaCl}$ concentrations, this species produced roughly no aerial mycelium and the colony looked very poor indicating the high stress conditions. Taken together these results suggest that the adaptation capacity to high $\mathrm{NaCl}$ conditions by the three ochratoxin A producing species reduces from $P$. nordicum to $P$. verrucosum and finally to $A$. carbonarius. 
Figure 1. Growth rate of $P$. verrucosum, $P$. nordicum and $A$. carbonarius after growth on YES medium substituted with increasing amounts of $\mathrm{NaCl}$ for five days (Aspergillus) and nine days (Penicillium) at $25^{\circ} \mathrm{C}$ in case of $P$. verrucosum and $P$. nordicum and at $30{ }^{\circ} \mathrm{C}$ in case of $A$. carbonarius. An amount of $10 \mu \mathrm{L}$ of a spore suspension of $10^{6}$ spores $/ \mathrm{mL}$ were centrally spotted on an agar plate and incubated. The colony diameters were measured after six and nine days, respectively.

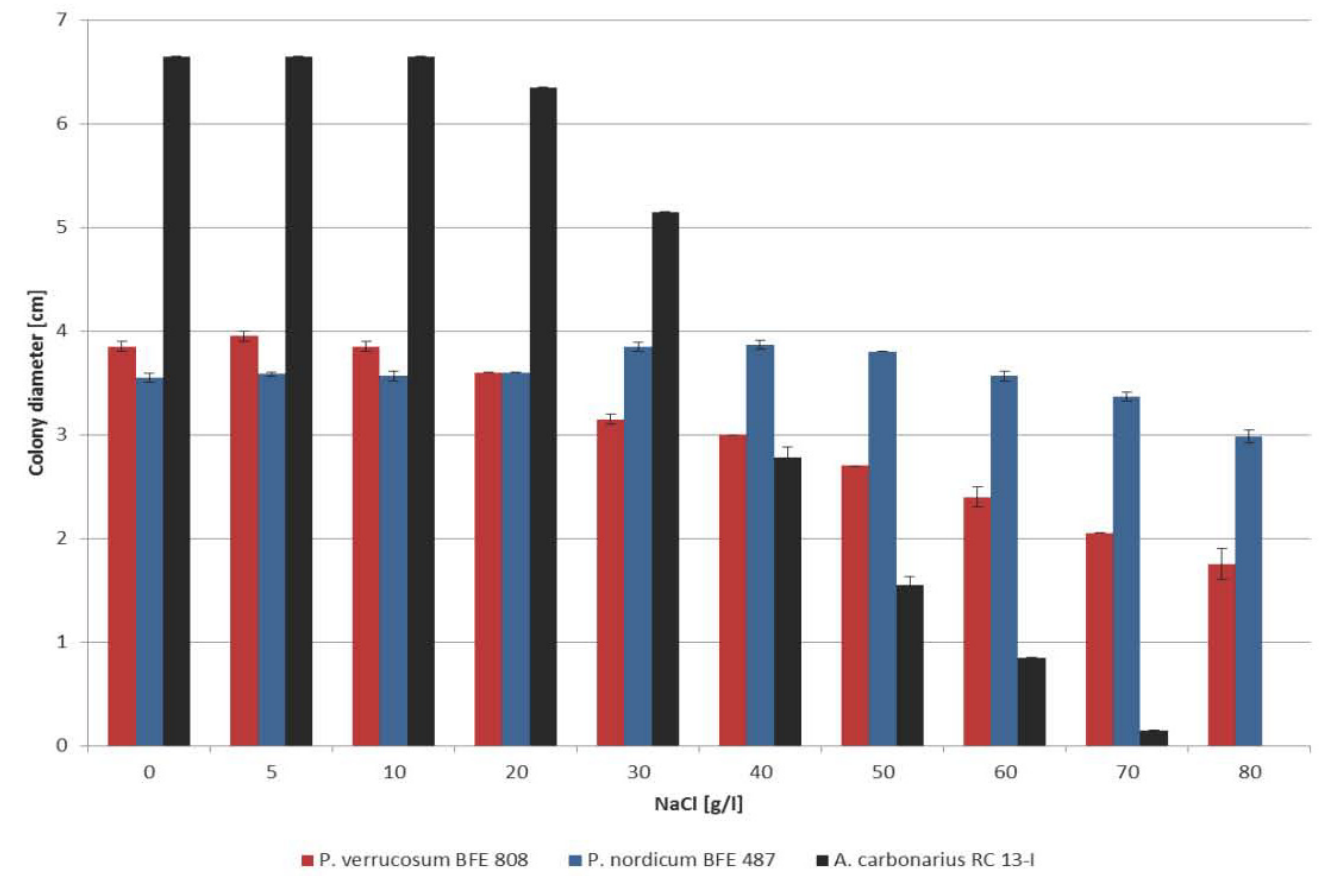

2.2. Different Correlations between HOG Phosphorylation and the Onset of Ochratoxin A Biosynthesis in the Three Species

Because of a possible relation between $\mathrm{NaCl}$ concentration, $\mathrm{HOG}$ phosphorylation and regulation of ochratoxin A biosynthesis, the dependence was analyzed in all three ochratoxin A producing species. For this purpose P. nordicum BFE487, P. verrucosum BFE808 and A. carbonarius RC13-I were grown on YES medium with increasing amounts of $\mathrm{NaCl}$. After nine days of incubation at $25{ }^{\circ} \mathrm{C}$ and $30{ }^{\circ} \mathrm{C}$ respectively, samples were withdrawn. One sample was taken to analyze the kinetics of ochratoxin A biosynthesis by HPLC. The other sample was taken for western blotting to visualize the $\mathrm{NaCl}$ dependent phosphorylation of HOG. Figure 2 shows the results.

The production of ochratoxin A by $P$. nordicum is consistent over a wide concentration range (Figure 2A). Also at high concentrations of $80 \mathrm{~g} / \mathrm{L} \mathrm{NaCl}$ high amounts of ochratoxin were produced. At concentrations below $20 \mathrm{~g} / \mathrm{L} \mathrm{NaCl}$ the amount of ochratoxin produced by this species is reduced. Interestingly the phosphorylation status of HOG follows that of the production of ochratoxin A in that the HOG is phosphorylated over the whole concentration range to a quite constant amount. Also in the control medium, e.g., in unsubstituted YES medium a phosphorylation can be detected and in fact also under these conditions ochratoxin was produced, indicating that YES per se activates the HOG pathway and leads to induction of ochratoxin A biosynthesis. The fact that at some $\mathrm{NaCl}$ concentrations $(20,40$ and $60 \mathrm{~g} / \mathrm{L} \mathrm{NaCl}) \mathrm{HOG}$ exhibits somewhat higher phosphorylation may be 
attributed to the fact, that the phosphorylation is not constant and quite flexible over time and only a snap shot is shown here. In case of $P$. verrucosum the situation is different (Figure 2B). As mentioned above this species is able to produce, beside ochratoxin A, the structurally very similar mycotoxin citrinin. In this case, as was already shown [15] citrinin is produced on YES medium at low concentrations of $\mathrm{NaCl}$. Beyond $40 \mathrm{~g} / \mathrm{L} \mathrm{NaCl}$ only ochratoxin and no citrinin at all is produced. Interestingly also in the case of $P$. verrucosum BFE808 the phosphorylation status of HOG follows that of ochratoxin A biosynthesis. In contrast to $P$. nordicum only at higher $\mathrm{NaCl}$ concentrations a reasonable phosphorylation of HOG becomes apparent. Especially at concentrations above $40 \mathrm{~g} / \mathrm{L}$, at conditions where only ochratoxin A and no citrinin is produced by the fungus, the phosphorylation of HOG increases with increasing concentrations of $\mathrm{NaCl}$.

Figure 2. Relation of the production of ochratoxin A and citrinin (in case of $P$. verrucosum) determined by HPLC with the phosphorylation status of the HOG protein, determined by western blotting for P. nordicum (A); P. verrucosum (B); and A. carbonarius $(\mathbf{C})$. The fungal strains were incubated on YES medium with increasing amounts of $\mathrm{NaCl}$ at $25{ }^{\circ} \mathrm{C}$ (Penicillia) or $30^{\circ} \mathrm{C}$ (Aspergillus) for nine days. After that time, samples were withdrawn, subjected to HPLC or to western blotting.

A
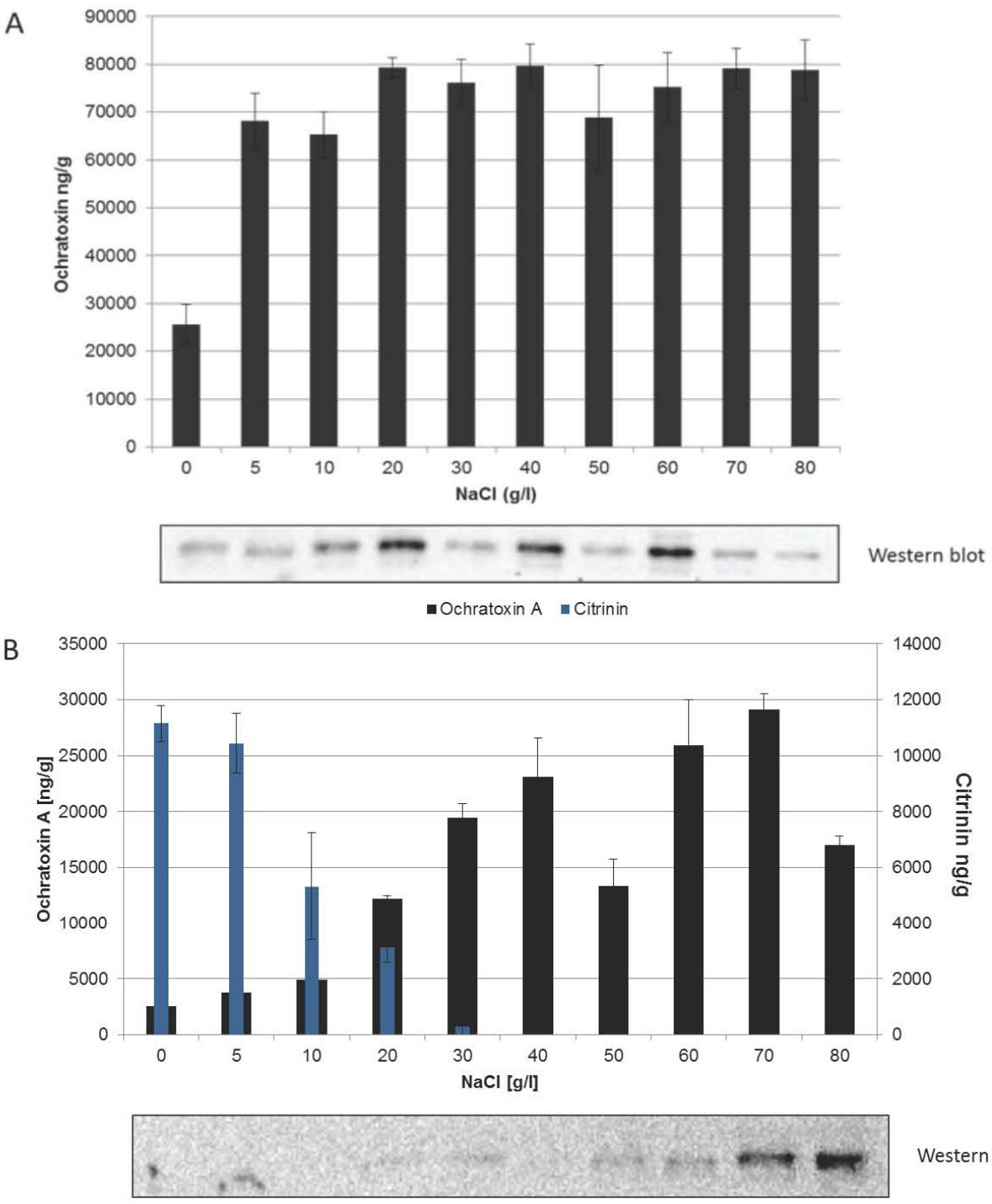
Figure 2. Cont.

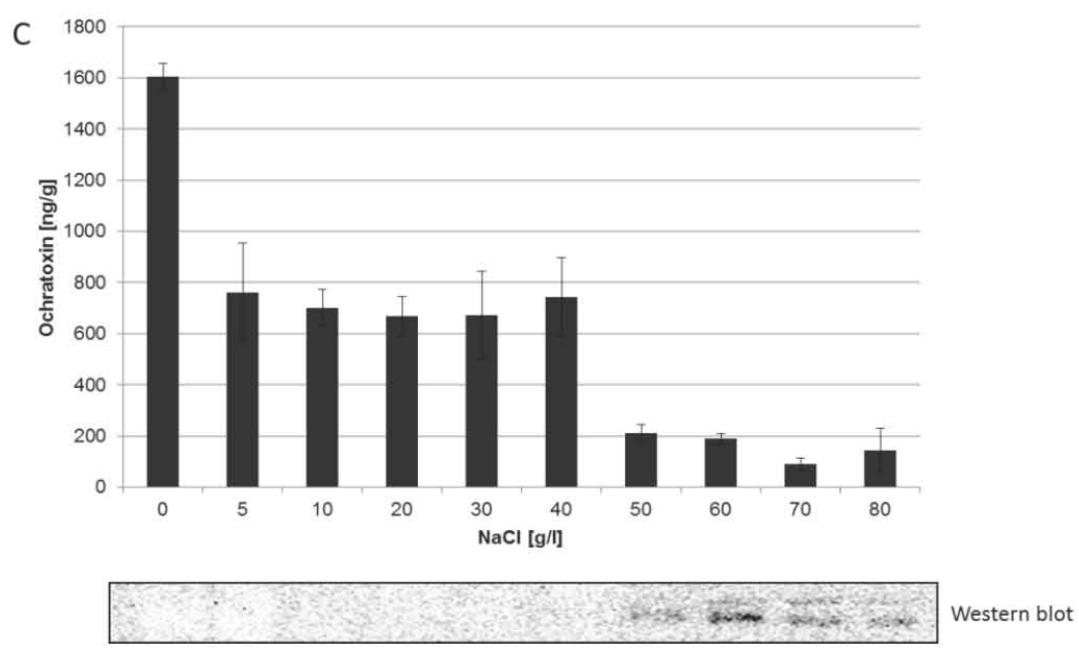

Taken together the situation in the two Penicillia strongly suggest a relation between HOG phosphorylation and the onset of ochratoxin A biosynthesis, which however is specific for each species.

The situation in the case of $A$. carbonarius is just opposite to that of the Penicillia (Figure $2 \mathrm{C}$ ). The phosphorylation status is nearly the same as that in $P$. verrucosum. Only at concentrations above $40 \mathrm{~g} / \mathrm{L}$ a phosphorylation of HOG becomes apparent. In case of $A$. carbonarius a double band arose after western blotting. Because of the fact that the p32 antibodies are usually specific for HOG, the result suggests that multiple forms of HOG exist in A. carbonarius (evtl. degradation products). Concerning the phosphorylation status the great difference between $A$. carbonarius and $P$. verrucosum is the fact that exactly at $\mathrm{NaCl}$ concentrations which lead to increased $\mathrm{HOG}$ phosphorylation the amount of ochratoxin A produced by A. carbonarius is drastically reduced. In parallel also the growth of the fungus is strongly inhibited under these conditions. This situation is not only true with A. carbonarius RC13-I, which is a moderate to strong ochratoxin A producing strain, but also with A. carbonarius ITEM5008, which in our hands is a low producing strain, indicating that this situation is typical for A. carbonarius (data with ITEM5008 not shown). The A carbonarius specific results indicate that HOG phosphorylation and ochratoxin A biosynthesis are obviously not coupled in this species in contrast to the situation in the two Penicillia. This means that increasing concentrations of $\mathrm{NaCl}$ does not lead to increasing concentrations of ochratoxin A.

\subsection{Influence of Increasing Amounts of Glucose on Growth, Ochratoxin A Biosynthesis and HOG Phosphorylation by $\mathrm{P}$. nordicum and A. carbonarius}

Because it was shown that $\mathrm{NaCl}$ as an osmotically active substance, which occurs in the natural habitat of the Penicillia, has a profound but differential effect on the regulation of the biosynthesis of ochratoxin A in the three species, the influence of glucose, a sugar which occurs in the habitat of A. carbonarius was analyzed. For this purpose the A. carbonarius RC13-I and P. nordicum BFE487 were grown on MEA medium with increasing amounts of glucose and growth was measured by determining the colony diameter, ochratoxin A biosynthesis by HPLC and the phosphorylation status of HOG by western blotting. MEA medium instead of YES medium was taken, because YES contains 
high amounts of sugar (sucrose) which might interfere with the added glucose. The growth behavior of P. nordicum and A. carbonarius are shown in Figure 3.

Figure 3. Growth rate of $A$. carbonarius and $P$. nordicum on malt glucose medium (MEA medium). The growth rate was determined as described in legend to Figure 1.

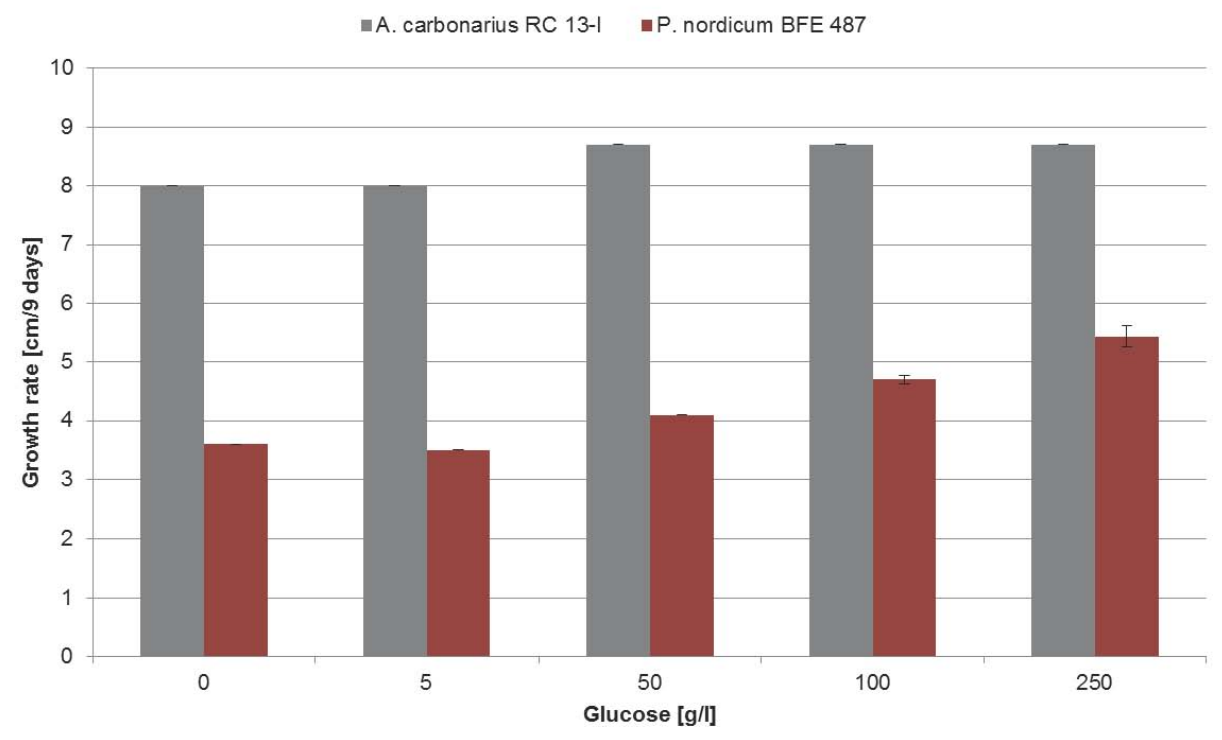

There was a tendency of increasing growth rates with increasing glucose concentrations up to the highest concentration $(250 \mathrm{~g} / \mathrm{L}$ glucose). These results indicate that in contrast to $\mathrm{NaCl}$, high concentrations of glucose are not growth inhibitory to both species.

Figure 4. Influence of the glucose concentration on the biosynthesis of ochratoxin $\mathrm{A}$ and on the phosphorylation status of HOG by P. nordicum (A); and A. carbonarius (B). The fungal strains were grown for nine days on MEA medium with increasing amounts of glucose. After that time, samples were withdrawn and used in HPLC to determine the ochratoxin A produced and for western blot analysis to visualize the phosphorylation status of HOG.
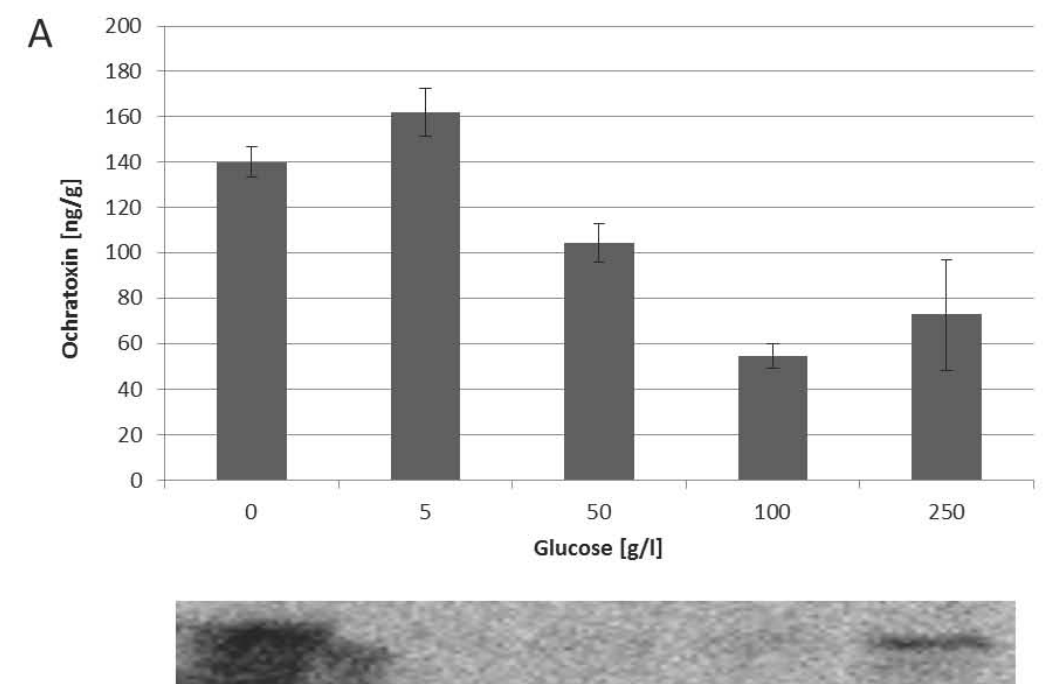
Figure 4. Cont.
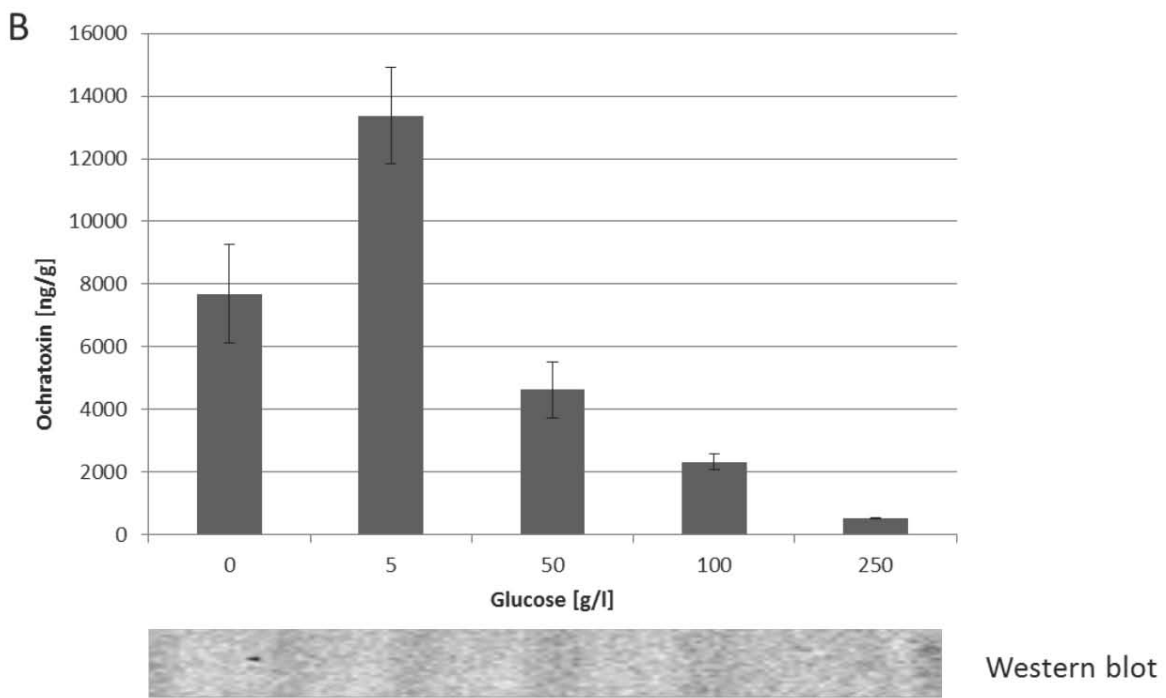

In contrast to the effect on growth, increasing amounts of glucose from 50 to $250 \mathrm{~g} / \mathrm{L}$ has an inhibitory influence on the biosynthesis of ochratoxin A in the case of P. nordicum (Figure 4A) and also in the case of $A$. carbonarius (Figure 4B).

This decrease is even stronger for A. carbonarius than for P. nordicum. For both species low concentrations of glucose $(5 \mathrm{~g} / \mathrm{L})$ lead to an increase in ochratoxin A biosynthesis.

Interestingly, albeit glucose has an influence on the osmotic status of the medium, it apparently does have only a weak influence on the phosphorylation of HOG. This is in sharp contrast to the situation with $\mathrm{NaCl}$. Under all glucose conditions analyzed no phosphorylation could be observed for A. carbonarius. In case of $P$. nordicum, a phosphorylation of HOG could only be observed at the highest glucose concentration $(250 \mathrm{~g} / \mathrm{L})$. Taken together the results suggest that in contrast to the osmotically active $\mathrm{NaCl}$ from the habitat of Penicillium, the osmotically active glucose from the habitat of $A$. carbonarius is not an inductor for ochratoxin A biosynthesis for the latter species.

\subsection{Dependence of the Biosynthesis of Ochratoxin A on Functional HOG}

It has been demonstrated in the previous experiments that the phosphorylation of the HOG MAP kinase is related to the biosynthesis of ochratoxin in the case of Penicillium. In order to analyze whether a functional HOG protein is indeed needed for the production of ochratoxin A under high osmolar conditions, an ATMT (Agrobacterium tumefaciens mediated transformation) was carried out with a vector containing the hygB gene between the left and right border of the T-DNA. The hygB gene itself was flanked by fragments of the $5^{\prime}$ respectively $3^{\prime}$ end of the $P$. verrucosum hog gene (Figure 5A). With this construct an A. tumefaciens mediated transformation was carried out. About 10 transformants were obtained. From these 4 transformants were further analyzed, but only the results of one is shown, which was taken for further analysis (transformant 4). The transformant was grown on YES medium (substituted with $80 \mathrm{~g} / \mathrm{L} \mathrm{NaCl}$ to induce ochratotoxin A biosynthesis) respectively MEA medium (MEA medium favors citrinin biosynthesis) containing hygromycin at $25{ }^{\circ} \mathrm{C}$. After 7 days samples were withdrawn and subjected to TLC (Figure 5B). As expected the wild type 
produced citrinin on MEA medium (Figure 5B, lane 3) and high amounts of ochratoxin on $\mathrm{NaCl}$ substituted YES medium (Figure 5B, lane 4). In contrast, the transformant produced no detectable amounts of ochratoxin (Figure 5B, lane 6) but normal amounts of citrinin (Figure 5B, lane 5). A subsequent Southern blot experiment in which a part of the hog gene was used as a probe revealed that the vector indeed integrated into the chromosomal hog gene, because the hybridization signal shifted to a higher position in the gel (Figure 5C). The visible multiple bands may be due to the homologous integration and further ectopic integrations.

Figure 5. Inactivation of the hog gene by ATMT. The cassette used for homologous integration (A) into the hog gene, was cloned in pPK2 [18]. LB, RB left and right border of the Ti DNA respectively, $\Delta h o g 5^{\prime}, \Delta h o g 3^{\prime}$ DNA regions from the upstream (5') and downstream (3') of the hog gene, hphB hygromycin resisstance gene from Streptomyces hygroscopicus. The vector was transformed into P. verrucosum BFE808 by A. tumefaciens mediated transformation. One of the resulting transformants (T4) was analyzed by TLC to visualize its ochratoxin A and citrinin production capacity $(\mathbf{B})$ and to compare it with the wild type (WT). Citrinin shows the typical smear, which is due to the high number of hydroxyl groups which interfere with the silica gel under the conditions used. Transformants with a changed capacity to produce ochratoxin A were further analyzed by Southern blotting (C). For Southern blotting the chromosomal DNA of the wild type and the transformant T4 were digested with EcoR1, separated on an agarose gel (the vertical arrow indicates the direction of electrophoresis), transferred to a nylon membrane and hybridized to the $h o g$ gene probe. In the transformant a shift of the hybridization signal towards higher fragment lengths can be seen $(\mathbf{C})$ indicating that the vector had integrated into the chromosomal hog gene and thereby had inactivated the gene.

A

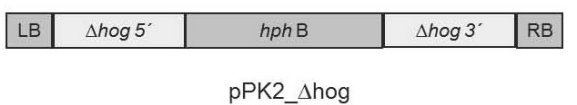

B

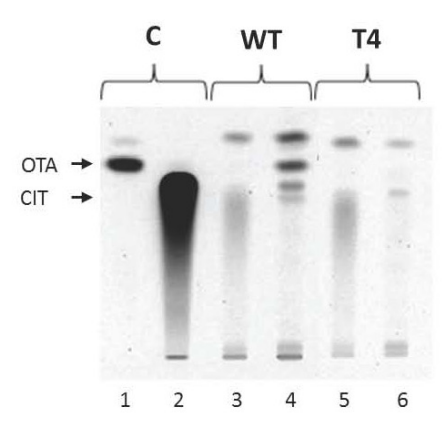

C

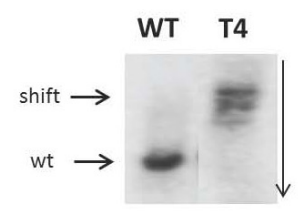


Taken together the results indicate that an inactivation of $h o g$ abolishes ochratoxin biosynthesis, but not citrinin biosynthesis in $P$. verrucosum, suggesting that ochratoxin biosynthesis is controlled by the hog MAP kinase, but not citrinin.

\section{Discussion}

The three ochratoxigenic species $P$. verrucosum, $P$. nordicum and $A$. carbonarius occur in completely different habitats. $P$. nordicum is adapted to $\mathrm{NaCl}$ rich environments like dry cured meats and cheeses [7,8]. P. verrucosum can mainly be found in cereals like wheat and is responsible for the production of ochratoxin in the commodity [7] and A. carbonarius is the predominant ochratoxigenic species on grapes and grape products [4,6]. A. carbonarius and $P$. nordicum do not show any known cross-occurrence, e.g., P. nordicum has never been reported to be found on grapes, nor A. carbonarius has ever been found on $\mathrm{NaCl}$ rich products. In contrast $P$. verrucosum seems to be much more adaptive with respect to the environment. Beside cereals it can occasionally be found also on $\mathrm{NaCl}$ rich products like dry cured meats or cheeses [11] or salted olives [12,13] but also occasionally on grapes [19]. It has been discussed earlier that this adaptation to different environments by $P$. verrucosum is paralleled by a shift in the production of secondary metabolites [15]. Under conditions, which impose oxidative stress, like for example under intense light conditions (field conditions), $P$. verrucosum shifts its secondary metabolite profile towards citrinin [14]. Citrinin is supposed to be a light protectant [20] and a potent antioxidant [21] and in fact, in certain cereal samples the contamination with citrinin was more pronounced than with ochratoxin A [22,23]. On the other hand, on substrates with increased concentrations of $\mathrm{NaCl}$, the secondary metabolite profile of P. verrucosum is shifted towards ochratoxin A [15]. It has been shown that the production of ochratoxin A under high $\mathrm{NaCl}$ conditions is an adaptive reaction [15]. High $\mathrm{NaCl}$ conditions not only decrease the $a_{\mathrm{w}}$ of the substrate, but the chloride ion has additional toxigenic potential, even at $a_{\mathrm{w}}$ values which have only limited inhibitory effects when caused by other osmolytes [24]. This is also demonstrated in the current work by the drastic reduction in the growth rate of $A$. carbonarius under high $\mathrm{NaCl}$ conditions, which was not observed under high glucose conditions, despite the fact that at the highest glucose concentration which did not influence the growth of $A$. carbonarius an $a_{\mathrm{w}}$ of roughly 0.96 was achieved. About the same $a_{\mathrm{w}}$ was achieved in the concentration range of $60-70 \mathrm{~g} / \mathrm{L}$ $\mathrm{NaCl}$ which drastically reduces the growth of $A$. carbonarius. Ochratoxin A carries a chlorine in its molecule and under high chloride ion concentrations, which are toxic for the cell [24,25] the permanent production and excretion of ochratoxin A leads to a permanent flow of chlorine out of the cell and ensures at least partial chloride homeostasis. This mechanism lends the ochratoxin A producing fungus a competitive advantage and a higher viability in $\mathrm{NaCl}$ rich environments.

Of course if ochratoxin A biosynthesis is important in $\mathrm{NaCl}$ rich environments, the fungus must be able to measure the external salt concentration and relate this to the regulation of ochratoxin $\mathrm{A}$ biosynthesis within the cell. Generally, external stimuli like changes in $\mathrm{pH}$, oxidative conditions or changes in the osmotic conditions are transmitted to the genetical level by signal transduction pathways. These signal transduction pathways translate the external signal to the transcriptional level by activating positive or negative transcription factors, which in turn regulates downstream genes. Fungi have various signal cascades, which are activated by different external signals [26]. The signal 
cascade, which is activated by high osmolarity in the medium, is the hog (high osmolarity glycerol) signal cascade [26]. This signal cascade consists of an osmosensor located in the membrane which transmits its signal via a two component system to a set of three consecutive protein kinases (MAP kinases), the last of which is the HOG protein kinase [26]. The phosphorylated form of this kinase is the active form and can activate downstream transcription factors by phosphorylation.

The involvement of the hog signal transduction pathway in the regulation of mycotoxins has been described for trichothecene biosynthesis in case of $F$. graminearum [16], for fumonisin biosynthesis in the case of $F$. proliferatum [27] and for alternariol biosynthesis in A. alternata [17]. Interestingly also in the case of $F$. graminearum and A. alternata $\mathrm{NaCl}$ plays an essential role in the regulation of the respective mycotoxins via HOG. In $P$. nordicum a paralleled activation of $\mathrm{HOG}$ phosphorylation and a coincident biosynthesis of ochratoxin A could be observed which suggest dependence between both processes. This dependence could be confirmed by the inactivation of the hog gene in P. verrucosum. The inactivated transformant was no longer able to produce ochratoxin A under high $\mathrm{NaCl}$ conditions in contrast to citrinin biosynthesis, which was not affected. That is in agreement with results from Li et al. [28] who showed that citrinin is regulated mainly by another signal cascade, in particular a G-protein/cAMP/PKA signal cascade.

After several repetitions of these experiments and after being aware that the phosphorylation status of HOG is flexible over time and dependent on the actual conditions, the following general picture could be achieved: $P$. nordicum, which is adapted to high salt and other extreme conditions [9,29] shows a consistent phosphorylation of $\mathrm{HOG}$ over all concentrations of $\mathrm{NaCl}$ analysed. This is paralleled by a consistently high production of ochratoxin A. The phosphorylation in P. verrucosum is not that consistent as in $P$. nordicum. Phosphorylation here occurs only at higher concentrations of $\mathrm{NaCl}$ which activates ochratoxin A biosynthesis and thereby represses citrinin biosynthesis. With this mechanism $P$. verrucosum is apparently able to change its adaptation from the cereal to the dry cured or salted food environment. In any case the results strongly suggest that in Penicillium the phosphorylation of HOG and the induction of ochratoxin A biosynthesis is coupled, which was also confirmed by gene inactivation in $P$. verrucosum. It has to be kept in mind however that because of the complexity of the regulation and the possible involvement of other regulatory mechanisms the phosphorylation of hog is an indication for the induction of the ochratoxin biosynthesis genes, but must not be related to the biosynthesis of ochratoxin A in a one to one ratio. Furthermore, it seems that phosphorylated HOG is not an absolute requirement for ochratoxin A biosynthesis, because ochratoxin A can also be synthesized when HOG phosphorylation is low or non-existing at all (for example under glucose conditions). This indicates that also other factors play a role in the regulation of ochratoxin A biosynthesis.

The mechanism described above, that the continuous production of ochratoxin A increases the viability of the producing organism in $\mathrm{NaCl}$ rich environments should theoretically be due to all ochratoxin A producing organisms. This is however not the case for A. carbonarius. Already at concentrations above $40 \mathrm{~g} / \mathrm{L}$ this species drastically loose the capacity to produce ochratoxin A and concomitantly the ability to grow. According to the results demonstrated here this is at least partly due to the fact that apparently HOG phosphorylation and activation of ochratoxin A biosynthesis is not coupled. As in P. verrucosum, $\mathrm{HOG}$ is phosphorylated at higher $\mathrm{NaCl}$ concentrations, but ochratoxin A biosynthesis in A. carbonarius only occurs at lower concentrations of $\mathrm{NaCl}$. Because of this 
uncoupling between HOG phosphorylation, which is a signal for challenging osmotic conditions, and the activation of ochratoxin A biosynthesis, A. carbonarius misses one mechanism to cope with high $\mathrm{NaCl}$ stress.

Because of the fact the $A$. carbonarius is adapted to a sugar rich environment, the influence of high concentrations of glucose on growth and ochratoxin A biosynthesis was analyzed. High concentrations of glucose did not negatively influence the growth of $A$. carbonarius and $P$. nordicum. However high concentration of glucose drastically reduces the production of ochratoxin $\mathrm{A}$, in $A$. carbonarius even more than in P. nordicum. Moreover, no glucose dependent phosphorylation of HOG could be observed in A. carbonarius. Only at the highest concentration was HOG phosphorylated in $P$. nordicum indicating that glucose induces $\mathrm{HOG}$ phosphorylation much less that $\mathrm{NaCl}$ in both species. That is in contrast to the situation in Saccharomyces cerevisiae [30], suggesting that the influence of glucose in filamentous fungi might be different.

\section{Experimental Section}

\subsection{Strains and Growth Conditions}

P. nordicum BFE487 is a strong ochratoxin A producing strain, P. verrucosum BFE808 is an ochratoxin A and citrinin producing strain. A. carbonarius RC13-I is a consistent but moderately ochratoxin A producing strain, whereas A. carbonarius ITEM 5008 only produces low amounts of ochratoxin A in our hands. These strains were used as model strains throughout this study. The strains were routinely grown on malt extract agar (Merck, Darmstadt, Germany) prepared according to the manufacturer's recommendations, except that $5 \mathrm{~g} / \mathrm{L}$ glucose were added (MEA). For ochratoxin A biosynthesis the cultures were either incubated on MEA or YES medium (yeast extract $20 \mathrm{~g} / \mathrm{L}$; sucrose $150 \mathrm{~g} / \mathrm{L}$; agar $20 \mathrm{~g} / \mathrm{L})$ which was supplemented with the respective amount of $\mathrm{NaCl}(0-100 \mathrm{~g} / \mathrm{L})$ for 5-7 days at $25{ }^{\circ} \mathrm{C}$ (Penicillia) or $30{ }^{\circ} \mathrm{C}$ (Aspergilli).

\subsection{Growth Assessment}

For analyzing the growth rate, the strains were single point inoculated on the agar plates and grown for 9 days under the respective conditions. For this purpose a suspension of $10^{6}$ spores per mL were prepared by harvesting spores of a seven day old colony with the aid of an inoculation loop and subsequent suspension in TWS solution ( $25 \mathrm{~mL}$ Tween 80 (1\% aqueous solution), $8 \mathrm{~g} \mathrm{NaCl}$ and $1 \mathrm{~L}$ distilled water). The spore number were counted in a Thoma chamber and adjusted by adding additional TWS solution if necessary. An amount of $10 \mu \mathrm{L}$ of that solution was centrally inoculated on an agar plate. After inoculation of the cultures the diameters of the colonies were measured two times rectangular to each other. All experiments were repeated three times.

\subsection{Determination of Ochratoxin A and Citrinin by Thin Layer (TLC) and High Pressure Liquid (HPLC) Chromatography}

For determination of ochratoxin $\mathrm{A} / \mathrm{B}$ and citrinin biosynthesis, an agar plug $(\varnothing 1 \mathrm{~cm})$ of the respective colony was taken from the region between center and edge of the colony with the aid of a sterile corer. This agar plug with the adhering mycelium was transferred into $2 \mathrm{~mL}$ micro-reaction 
tubes and $1 \mathrm{~mL}$ of chloroform was added. The fungal mycelia were extracted for $30 \mathrm{~min}$ at room temperature on a rotary shaker; the mycelia were discarded and the chloroform extract was evaporated to dryness in a vacuum concentrator (Speed Vac, Savant Instruments, Farmingdale, NY, USA). The extracts of the triplicates were merged for quantitative determination of ochratoxin A and citrinin on a Hitachi D-7000 HPLC system (Merck, Tokio, Japan) equipped with an auto-injector, column oven and fluorescence detector. The column oven was set to $40{ }^{\circ} \mathrm{C}$; the fluorescence detector was set to an excitation of $331 \mathrm{~nm}$ and an emission of $500 \mathrm{~nm}$. The flow rate was $0.7 \mathrm{~mL} / \mathrm{min}$ and the injection volume $10 \mu \mathrm{L}$. Solvent A consist of $250 \mathrm{mM}$ ortho-phosphoric acid and solvent B of methanol. Separation was carried out on a LiChrospher 100, C18 (250 mm, Ø $4 \mathrm{~mm}$ i.d., particle size $5 \mu \mathrm{m})$ reversed phase column (VWR International $\mathrm{GmbH}$, Darmstadt, Germany) using the following gradient: 0 min—solvent A 60\%, solvent B 40\%; $7 \mathrm{~min}-40 \%, 60 \% ; 12 \mathrm{~min}-35 \%, 65 \%$; $16 \mathrm{~min}-5 \%$, $95 \% ; 27 \mathrm{~min}-60 \%, 40 \%$. The limit of quantification was $25 \mathrm{pg}$ on column. Data collection and handling was done with EZ-Chrome Elite 3.2. All used standards were obtained from Sigma (Taufkirchen, Munich, Germany) with a purity of $\geq 98 \%$.

\subsection{Generation and Treatment of Data}

Each experiment was repeated at least three times. The mean and SD values for growth and toxin biosynthesis are shown. The western blots gave the same general trends several times.

\subsection{Protein Extraction}

After five days of incubation at $25^{\circ} \mathrm{C}$, the mycelium was scratched off the agar by the aid of a scalpel and $700 \mathrm{mg}$ were transferred directly into $1 \mathrm{~mL}$ protein extraction buffer $(1 \mathrm{M}$ Tris, $1 \mathrm{M}$ $\mathrm{MgCl}_{2}, 50 \mathrm{mM}$ EDTA, pH 7.5) supplemented with protease inhibitor (cOmplete Mini, EDTA-free Protease Inhibitor Cocktail, Roche, Mannheim, Germany) and phosphatase inhibitor (PhosSTOP, Roche, Mannheim, Germany). The proteins were extracted by repeated sonication for 5 minutes on ice. After spinning for $10 \mathrm{~min}$ at $4000 \mathrm{rpm} 200 \mu \mathrm{L}$ trichloracetic acid was added to the separated supernatant protein fraction. The samples were cooled on ice for $30 \mathrm{~min}$ and spun for $7 \mathrm{~min}$ at 13,000 $\mathrm{rpm}$. The protein pellet was resuspended in $150 \mu \mathrm{L}$ Laemmli-buffer $(0.5 \mathrm{M}$ Tris, $192 \mathrm{mM}$ SDS, 20\% glycerol, $10 \% \beta$-mercaptoethanol and bromphenol blue) and evaporated with ammonia gas to yield deprotonated proteins. The protein extracts were denatured at $95{ }^{\circ} \mathrm{C}$ and stored at $-80{ }^{\circ} \mathrm{C}$ until further analysis.

\subsection{SDS PAGE and Western Blot}

Proteins were separated by SDS PAGE according to Sambrook and Russel [31] and the gel was stained with Coomassie brilliant blue. The relative protein concentration was determined with a Biorad Chemidoc XRS Imaging System (Biorad, Gaithersburg, MD, USA) and the samples were adjusted to equivalent concentrations. The same protein amounts for each sample were loaded onto a new gel and the separated proteins were transferred onto a nylon membrane. The treatment with the p38 antibody specific for phosphorylated HOG (Cell Signaling, Danvers, MA, USA) was performed essentially as described by the manufacturer of the labeling kit. After rinsing and drying, the membrane was 
incubated with $10 \mathrm{~mL}$ LumiGLO solution (Cell Signaling, Danvers, MA, USA). The luminescence was detected with a Biorad Chemidoc XRS Imaging System (Biorad, Gaithersburg, MD, USA). The resulting band intensities after luminescence measuring were evaluated visually.

\subsection{A. tumefaciens Mediated Transformation (ATMT) to Inactivate the hog Gene}

DNA Fragments covering about 500 bp of the 5' and the $3^{\prime}$ end of the $P$. verrucosum hog gene were amplified by PCR and cloned upstream and downstream of the hygromycin B resistance gene between the right and left border of the binary vector pPK2 [18]. The resulting disruption cassette is shown in Figure 2A. A. tumefaciens AGL-1 cells which carried the vector pPK2 containing the disruption cassette were grown at $25{ }^{\circ} \mathrm{C}$ for $48 \mathrm{~h}$ in minimal medium which was supplemented with kanamycin $\left(50 \mu \mathrm{g} \mathrm{mL}^{-1}\right)$. The cells were then diluted reaching an $\mathrm{OD}_{660}$ of 0.15 in induction medium (IM) (10 mmol/L K $\mathrm{HPO}_{4}, 10 \mathrm{mmol} / \mathrm{L} \mathrm{KH}_{2} \mathrm{PO}_{4}, 2.5 \mathrm{mmol} / \mathrm{L} \mathrm{NaCl}, 2 \mathrm{mmol} / \mathrm{L} \mathrm{MgSO}_{4}, 0.7 \mathrm{mmol} / \mathrm{L} \mathrm{CaCl}_{2}, 9$ $\mathrm{mmol} / \mathrm{L} \mathrm{FeSO}_{4}, 4 \mathrm{mmol} / \mathrm{L}\left(\mathrm{NH}_{4}\right)_{2} \mathrm{SO}_{4}, 10 \mathrm{mmol} / \mathrm{L}$ glucose, $40 \mathrm{mmol} / \mathrm{L}$ 2-[N-morpholino] ethan sulfonic acid, $\mathrm{pH} 5.3$, and $0.5 \%$ glycerol) in the presence of $200 \mu \mathrm{mol} / \mathrm{L}$ acetosyringone (AS). The cells were further grown for $9 \mathrm{~h}$ before mixing with an equal volume of a spore suspension from $P$. verrucosum BFE808. This solution was plated onto cellophane sheets which were placed on the agar plates containing the co-cultivation medium (IM + AS supplemented with $5 \mathrm{mmol} / \mathrm{L}$ instead of $10 \mathrm{mmol} / \mathrm{L}$ of glucose). After the co-cultivation procedure at $25{ }^{\circ} \mathrm{C}$ for $36 \mathrm{~h}$, the cellophane sheets were transferred to $\mathrm{M}-100$ plates $\left(55 \mathrm{mmol} / \mathrm{L}\right.$ glucose and $30 \mathrm{mmol} / \mathrm{L} \mathrm{KNO}_{3}$ ) plus mineral solution (117 mmol/L KH $\mathrm{PO}_{4}, 28 \mathrm{mmol} / \mathrm{L} \mathrm{Na}_{2} \mathrm{SO}_{4}, 107 \mathrm{mmol} / \mathrm{L} \mathrm{KCl}, 8 \mathrm{mmol} / \mathrm{L} \mathrm{MgSO} 4 \times 7 \mathrm{H}_{2} \mathrm{O}, 9 \mathrm{mmol} / \mathrm{L}$ $\mathrm{CaCl}_{2}, 7.8 \mathrm{mmol} / \mathrm{L} \mathrm{H}_{3} \mathrm{BO}_{3}, \quad 5.6 \mu \mathrm{mol} / \mathrm{L} \quad \mathrm{MnCl}_{2} \times 4 \quad \mathrm{H}_{2} \mathrm{O}, 2.3 \mu \mathrm{mol} / \mathrm{L} \quad \mathrm{ZnCl}_{2}, 1.3 \mu \mathrm{mol} / \mathrm{L}$ $\mathrm{Na}_{2} \mathrm{MnO}_{4} \times 2 \mathrm{H}_{2} \mathrm{O}, 2.9 \mu \mathrm{mol} / \mathrm{L} \mathrm{FeCl}_{3} \times 6 \mathrm{H}_{2} \mathrm{O}$, and $\left.12.8 \mu \mathrm{mol} / \mathrm{L} \mathrm{CuSO}_{4} \times 5 \mathrm{H}_{2} \mathrm{O}\right)$ and $1.5 \%$ agar supplemented with hygromycin $\mathrm{B}(100 \mu \mathrm{g} / \mathrm{mL})$ as selection medium for fungal transformants and cefoxitin $(150 \mu \mathrm{g} / \mathrm{mL})$ to inhibit the growth of $A$. tumefaciens cells. After incubation for 10 days at $25^{\circ} \mathrm{C}$, the number of hygromycin B-resistant colonies was counted and analyzed further.

\subsection{Southern Blotting}

For Southern blotting experiments the EcoRI restriction enzyme digested DNA was separated on a $0.8 \%$ agarose gel and transferred onto a nylon membrane filter according to the method of Southern [32]. Hybridization was accomplished under stringent conditions as described by Sambrook and Russel [30]). The 1621 bp hog was labeled in a PCR reaction using the primers HOG_Pdigitatum_kpl_for (5'-ATGGCGGAATTCGTGCGTG-3') and HOG_Pdigitatum_kpl_rev (5'-TTATGCGAATGCTTGTCCAGTAGC-3'). The nucleotide mixture contained 11-DIG-UTP. Hybridization and staining of the filter were performed according to the recommendations of the manufacturer of the DIG-UTP Labelling and Detection kit (Boehringer, Mannheim, Germany).

\section{Conclusions}

Taken together, the ochratoxin A biosynthesis in Penicillia is $\mathrm{NaCl}$ concentration dependent and regulated via the phosphorylation of HOG. The constant high production of ochratoxin A by $P$. nordicum, ensured by a consistent phosphorylation status of $\mathrm{HOG}$, renders $P$. nordicum competitive 
under high salt conditions. The shift from citrinin towards ochratoxin A biosynthesis in P. verrucosum under high $\mathrm{NaCl}$ conditions, which is also regulated by $\mathrm{HOG}$ phosphorylation, make this cereal adapted organism partly competitive on substrates with high $\mathrm{NaCl}$ conditions and finally the uncoupling of ochratoxin A biosynthesis and HOG phosphorylation in A. carbonarius is apparently one of the reasons why this species is not adapted to $\mathrm{NaCl}$ environments. Therefore, one of the ecological reasons for ochratoxin A biosynthesis in Penicillium is apparently the maintenance of chloride homeostasis [15]. This is not the case in A. carbonarius. The ecological reason for the production of ochratoxin A by A. carbonarius must be different. Glucose (or other sugars) as a typical osmolytic active constituent of its natural habitat only has a negative influence of the biosynthesis of ochratoxin A. Therefore, a reasonable ecological reason for the biosynthesis of ochratoxin A by $A$. carbonarius cannot yet be deduced.

\section{Acknowledgments}

This work was financed by the EU-Project MycoRed EC KBBE-2007-222690-2 MYCORED. We thank Giancarlo Perrone for the A. carbonarius strain ITEM5008 and Lorena Ponsone/Sofia Chulze for A. carbonarius RC13-I.

\section{Conflict of Interest}

The authors declare no conflict of interest.

\section{References}

1. Pardo, E.; Marin, S.; Ramos, A.J.; Sanchis, V. Occurrence of ochratoxingenic fungi and ochratoxin A in green coffee from different origins. Food Sci. Technol. 2004, 10, 45-49.

2. Palumbo, J.D.; O'Keefe, T.L.; Vasquez, S.J.; Mahoney, N.E. Isolation and identification of ochratoxin A producing Aspergillus section Nigri strains from Californian raisins. Lett. Appl. Microbiol. 2011, 52, 330-336.

3. Pitt, J.I.; Hocking, A.D. Fungi and Food Spoilage; Aspen Publishers: Gaithersburg, MD, USA, 1999.

4. Leong, S.I.L.; Hocking, A.D.; Scott, E.S. Effect of temperature and water activity on growth and ochratoxin A production by Australian Aspergillus carbonarius and A. niger isolates on a simulated grape juice medium. Int. J. Food Microbiol. 2006, 110, 209-216.

5. Battilani, P.; Giorni, P.; Pietri, A. Epidemiology of toxin-producing fungi and ochratoxin A occurrence in grape. Eur. J. Plant Pathol. 2003, 109, 715-722.

6. Perrone, G.; Mulè, G.; Susca, A.; Battilani, P.; Pietri, A.; Logrieco, A. Ochratoxin A Production and amplified fragment length polymorphism analysis of Aspergillus carbonarius, Aspergillus tubingensis, and Aspergillus niger strains Isolated from grapes in Italy. Appl. Environ. Microbiol. 2006, 72, 680-685.

7. Lund, F.; Frisvad; J.C. Penicillium verrucosum in wheat and barley indicates presence of ochratoxin A. J. Appl. Microbiol. 2003, 95, 1117-1123. 
8. Larsen, T.O.; Svendsen, A.; Smedsgaard, J. Biochemical characterization of ochratoxin A-producing strains of the genus Penicillium. Appl. Environ. Microbiol. 2001, 67, 3630-3635.

9. Sonjak, S.; Licen, M.; Frisvad, J.C.; Gunde-Cimerman, N. Salting of dry-cured meat-A potential cause of contamintation with the ochratoxin A-producing species Penicillium nordicum. Food Microbiol. 2011, 28, 1111-1116.

10. Butinar, L.; Frisvad, J.C.; Gunde-Cimerman, N. Hypersaline waters-A potential source of foodborne toxigenic Aspergilli and Penicillia. FEMS Microbiol. Ecol. 2011, 77, 186-199.

11. Peintner, U.; Geiger, J.; Pöder, R. The mycobiota of speck, a traditional tyrolean smoked and cured ham. J. Food Protect. 2000, 63, 1399-1403.

12. Heperkan, D.; Dazkir, G.S.; Kansu, D.Z.; Güler, F.K. Influence of temperature on citrinin accumulation by Penicillium citrinum and Penicillium verrucosum in black table olives. Toxin Rev. 2009, 28, 180-186.

13. Tokusoglu, Ö.; Bozoglu, F. Citrinin risk in black and green table olives: Simultaneous determination with ochratoxin-A by optimized extration and IAC-HPLC-FD. Ital. J. Food Sci. 2010, 22, 284-291.

14. Schmidt-Heydt, M.; Rüfer, C.E.; Raupp, F.; Bruchmann, A.; Perrone, G.; Geisen, R. Influence of light on food relevant fungi with emphasis on ochratoxin producing species. Int. J. Food Microbiol. 2011, 145, 229-237.

15. Schmidt-Heydt, M.; Graf, E.; Stoll, D.; Geisen, R. The biosynthesis of ochratoxin A by Penicillium as one mechanism for adaptation to $\mathrm{NaCl}$ rich foods. Food Microbiol. 2012, 29, 233-241.

16. Ochiai, N.; Tokai, T.; Nishiuchi, T.; Takahashi-Ando, N.; Fujimura, M.; Kimura, M. Involvement of the osmosensor histidine kinase and osmotic stress-activated protein kinases in the regulation of secondary metabolism in Fusarium graminearum. Biochem. Biophys. Res. Commun. 2007, 363, 639-644.

17. Graf, E.; Schmidt-Heydt, M.; Geisen, R. HOG MAP kinase regulation of alternariol biosynthesis in Alternaria alternata is important for substrate colonization. Int. J. Food Microbiol. 2012, 157, 353-359.

18. Covert, S.F.; Kapoor, P.; Lee, M.-H.; Briley, A.; Nairn, C.J. Agrobacterium tumefaciens-mediated transformation of Fusarium circinatum. Mycol. Res. 2001, 105, 259-264.

19. Bragulat, M.R.; Abarca, M.L.; Cabanes, J. Low occurrence of patulin- and citrinin-producing species isolated from grapes. Lett. Appl. Microbiol. 2008, 47, 286-289.

20. Stormer, F.C.; Sandven, P.; Huitfeldt, S.; Eduard, W.; Skogstad, A. Does the mycotoxin citrinin function as a sun protectant in conidia from Penicillium verrucosum? Mycopathologia 1998, 142, 43-47.

21. Heider, E.M.; Harper, J.K.; Grant, D.M.; Hoffmann, A.; Dugan, F.; Tomer, D.P.; O’Neil, K.O. Exploring unusual antioxidant activity in a benzoic acid derivative: A proposed mechanism for citrinin. Tetrahedron 2012, 62, 1199-1208.

22. Meister, U. New method of citrinin determination by HPLC after polyamide column clean-up. Eur. Food Res. Technol. 2004, 218, 394-399. 
23. Molinié, A.; Faucet, V.; Castegnaro, M.; Pfohl-Leszkowicz, A. Analysis of some breakfeast cereals on the French market for their contents of ochatoxin A, citrinin and fumonisin B1: Development of a method for simultaneous extraction of ochratoxin A and citrinin. Food Chem. 2005, 92, 391-400.

24. Samapundo, S.; Deschuyffeleer, N.; van Laere, D.; de Leyn, I.; Devlieghere, F. Effect of $\mathrm{NaCl}$ reduction and replacement on the growth of fungi important to the spoilage of bread. Food Microbiol. 2010, 27, 749-756.

25. Ayodele, S.M.; Ojoghoro, O.J. Salt tress effects on the vegetative growth of Pleurotus tuberregium (FR) sing. J. Biol. Sci. 2007, 7, 1278-1281.

26. Rispail, N.; Soanes, D.M.; Ant, C.; Czajkowski, R.; Grünler, A.; Huguet, R.; Perez-Nadales, E.; Poli, A.; Sartorel, E.; Valiante, V.; et al. Comparative genomics of MAP kinase and calcium-calcineurin signalling compontents in plant and human pathogenic fungi. Fungal Genet. Biol. 2009, 46, 287-298.

27. Kohut, G.; Ádám, A.L.; Fazekas, B.; Hornok, L. N-starvation stress induced FUM gene expression and fumonisin production is mediated via the HOG-type MAPK pathway in Fusarium proliferatum. Int. J. Food Microbiol. 2009, 130, 65-69.

28. Li, L.; Shao, Y.; Li, Q.; Yang, S.; Chen, F. Identification of Mga1, a G-protein alpha-subunit gene involved in regulating citrinin and pigment production in Monascus ruber M7. FEMS Microbiol. Lett. 2010, 308,108-114.

29. Gunde-Cimerman, N.; Sonjak, S.; Zalar, P.; Frisvad, J.C.; Diderichsen, B.; Plemenitas, A. Extremophilic fungi in arctic ice: A relationship between adaptation to low temperature and water activity. Phys. Chem. Earth 2003, 28, 1273-1278.

30. Jiménez-Martín, E.; Zuzuarregui, A.; Gomar-Alba, M.; Guteérrez, D.; Gil, C.; del Olmo, M. Molecular response of Saccharomyces cerevisiae wine and laboratory strains to high sugar stress conditions. Int. J. Food Microbiol. 2011, 145, 211-220.

31. Sambrook, J.; Russel, D.W. Molecular Cloning, 3rd ed.; Cold Spring Harbor Laboratory Press: Cold Spring Harbour, NY, USA, 2001.

32. Southern, E. Detection of specific sequences among DNA fragments separated by gel electrophoresis. J. Mol. Biol. 1975, 98, 503-517.

(C) 2013 by the authors; licensee MDPI, Basel, Switzerland. This article is an open access article distributed under the terms and conditions of the Creative Commons Attribution license (http://creativecommons.org/Licenses/by/3.0/). 